\title{
Interactive comment on "Coordination and \\ Control: Limits in Standard Representations of Multi-Reservoir Operations in Hydrological Modeling" by Charles Rougé et al.
}

\section{Anonymous Referee \#1}

Received and published: 10 January 2020

This study deploys the Method of Morris to evaluate the sensitivity of release and storage time series to adjustments in the parameters of generic reservoir operating rules. The analysis is performed using a high resolution hydrological model (WBM) and focuses on a cascade of reservoirs located in the Upper Snake River Basin. The paper is well written, with clearly defined methodology and easy-to-follow results section. The experiments conducted are ill-suited to the aims of the study, leaving too many confounding factors. Specifically, I disagree with the authors key claim that the approach exposes coordination between reservoirs missing from the generic operating schemes (see specific comments below). While I do not doubt that coordination does occur in this particular cascade system, I cannot see how this sensitivity analysis exposes 
that coordination unequivocally. I also have some concerns with the study design, and in particular the use of the high-resolution hydrological model, which introduces a severe and unnecessary computational constraint while also supplying the reservoirs with (likely) inaccurate historical inflows. The latter leaves the reader unsure as to whether the difference between simulated and observed storage/release is more a function of erroneous inflow than inaccurate operating decisions. This is particularly important when demonstrating the inadequacy of the reservoirs rules in representing flood and drought (it could simply be that the upstream hydrology from the model is delivering the wrong volumes of water). Since the actual observed inflows are available for this system, it would seem far more prudent to develop a simple, offline cascade model. I suspect such a radical change to the experimentation at this stage would be unrealistic, so I would instead encourage the authors to change the aims and storyline offered here. The simulations are sound and there is a great need for the community to learn more about the nature and performance of generic reservoir schemes. I would support a revision if either (a) the authors can convincingly rebut my concerns listed below, or (b) a new angle is developed with a more defensible conclusion.

Specific comments to authors:

Section 3.2. The justification for the $10 \%$ decision is unconvincing. Suppose a dam has an average inflow of 100 cumecs. An Rmin of 0.1 would be 10 cumecs and would vary by a maximum of 1 cumec. So the left-hand side of the operating curve hardly moves. In contrast, if your Rmax is 5 (=500 cumecs) then you'd vary this parameter by plus or minus 50 cumecs. So the right hand side of the curve will shift wildly in comparison. This is surely why Rmin appears to be unimportant in your sensitivity analysis; you've barely moved it. I appreciate that there are physical reasons why Rmin would be expected to be somewhat less variable than Rmax in absolute terms (although both are highly uncertain), but the uniform 10\% assumption is not ideal either, and it invalidates your later statements about which parameters constitute the signature of parametric influence (page 15). 
Section 4.2 through 4.4. The results described here show that the operations of upstream dams can affect the decisions made at downstream dams. This occurs because any change upstream has an effect on the inflows into a downstream dam, thus affecting its storage levels and therefore its releases (which are a function of storage). Sensitivity analysis exposes how the decisions taken at one dam are affected by the rules deployed at other dams (often with some intriguing complexities). This is insufficient, however, to claim that the models must be missing *coordination* (which presumably means dam operator A looking at the storage levels of dams $B$ and $C$ to inform his or her decision). Similarly, the results that describe inadequate representation of flood and drought mitigation "could* be caused by uncoordinated reservoir schemes. But inadequate mitigation could also be simply because the operating schemes at individual dams are insufficient. It's possible that more realistic operating schemes at the individual dams (i.e., not necessarily incorporating coordination, but with more realistic structure and/or parameterization) would provide the correct mitigation responses. It's also possible that the failure of these models to represent flood and drought mitigation is partly caused by bias in your inflow data (which is not shown or compared to observed at the upstream dam). Given these possibilities, the proposed framework fails to demonstrate unequivocally that a missing piece of the reservoir model is coordination.

Other minor comments:

Figure 1. Difficult to interpret due to color scheme used.

Table 1. Please provide some additional justification for these parameters. Has there been any validation done? Why would the Rref for a water supply reservoir be 0.1 ? This parameter should vary widely across water supply reservoirs as a function of demand relative to inflow (and 0.1 is very low). Generally I don't see why the purpose of the reservoir would control these parameters. Rmin (and indeed the whole left-hand section of the operating curve) would likely be strongly determined by environmental flow requirements, which are independent of reservoir purpose. I would also suggest

Printer-friendly version

Discussion paper
Interactive

comment 
discussing the omission of seasonality in the operational parameters (which could apply to non-irrigation reservoirs). If there is no good justification for these rules then this is ok (it's not the purpose of your experiment to defend the status quo)âĂ instance just make a statement to inform the reader.

P6 line 1 - reservoir data *were* derived...

P6 line 1 - not clear who performed these manual updates

P6 line 7 - How accurate are these demand data compared to USGS estimates, which are US specific.

Section 2.1. I hadn't studied the Method of Morris previously, but this is an excellent description that educates the reader.

P16 line 7 - The high variability in release (relative to observed) is surely just caused by the right-hand side slope of the operating curve being too steep, or the Sref being too low. Why should this point to incorrect representation of coordination?

P16 line 27 - this may have been a low flow event, but your figure for Jackson shows clearly that the drawdown was in large part caused by sustained high release through 2013.

It would be helpful to see inflow, storage, and release graphs for the full time series for all reservoirs (perhaps in supplemental material).

Interactive comment on Hydrol. Earth Syst. Sci. Discuss., https://doi.org/10.5194/hess-2019589, 2019. 